\title{
Decompressive craniectomy in a rat model of "malignant" cerebral hemispheric stroke: experimental support for an aggressive therapeutic approach
}

\author{
Arnd Doerfler, M.D., Michael Forsting, M.D., Wolfgang Reith, M.D., Christian Staff, Sabine \\ Heiland, Ph.D., Wolf-Rüdiger Schäbitz, M.D., Rüdiger von Kummer, M.D., Werner Hacke, M.D., \\ and Klaus Sartor, M.D.
}

Departments of Neuroradiology and Neurology, University of Heidelberg Medical School, Heidelberg, Germany

Acute ischemia in the complete territory of the carotid artery may lead to massive cerebral edema with raised intracranial pressure and progression to coma and death due to uncal, cingulate, or tonsillar herniation. Although clinical data suggest that patients benefit from undergoing decompressive surgery for acute ischemia, little data about the effect of this procedure on experimental ischemia are available. In this article the authors present results of an experimental study on the effects of decompressive craniectomy performed at various time points after endovascular middle cerebral artery (MCA) occlusion in rats.

Focal cerebral ischemia was induced in 68 rats using an endovascular occlusion technique focused on the MCA. Decompressive cranioectomy was performed in 48 animals (in groups of 12 rats each) 4, 12, 24, or 36 hours after vessel occlusion. Twenty animals (control group) were not treated by decompression craniectomy. The authors used the infarct volume and neurological performance at Day 7 as study endpoints.

Although the mortality rate in the untreated group was $35 \%$, none of the animals treated by decompressive craniectomy died (mortality $0 \%$ ). Neurological behavior was significantly better in all animals treated by decompressive craniectomy, regardless of whether they were treated early or late. Neurological behavior and infarction size were significantly better in animals treated very early by decompressive craniectomy (4 hours) after endovascular MCA occlusion (p less than 0.01 ); surgery performed at later time points did not significantly reduce infarction size.

The results suggest that use of decompressive craniectomy in treating cerebral ischemia reduces mortality and significantly improves outcome. If performed early after vessel occlusion, it also significantly reduces infarction size. By performing decompressive craniectomy neurosurgeons will play a major role in the management of stroke patients.

Key Words * cerebral infarction * craniectomy * experimental ischemia * malignant middle cerebral artery infarction * rat 
Ischemic cerebrovascular disease is the most frequent disease of the brain and may lead to death in about $15 \%$ of all stroke patients. Acute ischemia in the territory of the middle cerebral artery (MCA) can lead to massive cerebral edema with raised intracranial pressure and progression to coma and death. This phenomenon, known as "malignant MCA infarction" has been well documented in clinical observations and in autopsy studies.[5]

Although experimental evaluation of various antiedema agents, such as osmodiuretics, barbiturates, or Tris buffer, has indicated their beneficial effects in the treatment of ischemia-induced brain edema, no treatment has been shown to be consistently effective in the clinical setting.[13,33] Until recently, there has been no proven therapy for acute infarction, although restoration of cerebral blood flow by thrombolysis if started early has been shown to be effective in clearly defined subgroups of patients.[12,25] In stroke victims with massive unilateral hemispheric edema, who deteriorate despite maximum medical therapy, surgical decompression may be an effective therapeutic alternative. Although this kind of therapy is well established for treatment of large cerebellar infarcts, $[3,4,15,31]$ there exist only retrospective, anecdotal case reports of patients treated by decompressive craniectomy for supratentorial ischemia. $[8,11,16,17,19,28,30,39]$ Recently, the initial results of an open, prospective trial to determine the effects of decompressive craniectomy on mortality and morbidity have been reported.[32]

Thus far, little experimental data have been published on the usefulness of decompressive craniectomy in acute supratentorial stroke. A recently published experimental study in rats did show that decompressive craniectomy for cerebral ischemia reduced mortality and also significantly improved the clinical outcome in a time-dependent fashion.[10] However, in that study decompressive craniectomy was performed only at 1 and 24 hours after experimental MCA occlusion. One of the key factors determining the success of craniectomy is appropriate timing; however, the best point of time for craniectomy is still under debate. Therefore, we conducted a study in which we used an endovascular model for MCA occlusion in rats, to evaluate the effects of decompressive craniectomy on mortality, infarction size, and neurological outcome at different points of time after vessel occlusion.

\section{MATERIAL AND METHODS}

\section{Animal Preparation}

Focal cerebral ischemia was induced in 68 male Wistar rats, weighing 270 to $320 \mathrm{~g}$, using the intraluminal suture occlusion method first described by Koizumi and associates.[18] The study was approved by the local animal protection committee. The rats were allowed free access to food and water before the procedure and then anesthetized with a preparation of ketamine $(4 \mathrm{mg} / 100 \mathrm{~g})$ and xylazine $(1.5 \mathrm{mg} / 100 \mathrm{~g})$ administered intramuscularly. Monitoring of hematocrit, $\mathrm{pH}, \mathrm{PO} 2$ and PCO2 and blood pressure during surgery was performed using a femoral artery catheter. Rectal temperature was maintained at $37 \check{\mathrm{s} C}$ with a feedback-regulated heating pad throughout the operation.

In all animals the right MCA was occluded via a transvascular approach as previously described in detail.[18] For MCA occlusion, the right common carotid artery (CCA) and the right external carotid artery (ECA) were exposed through a midline neck incision. The distal CCA and the ECA were first ligated with a No. 4-0 suture. Then a No. 4-0 monofilament nylon suture (length $40 \mathrm{~mm}$ ), whose tip had been coated with silicone, was inserted through an arteriotomy of the CCA and gently advanced into the internal carotid artery (ICA) to a point approximately $17 \mathrm{~mm}$ distal to the carotid bifurcation. Mild resistance to this advancement indicated that the suture had entered the anterior cerebral artery (ACA), 
thus occluding the origins of the MCA and the posterior communicating artery. To prevent bleeding, the CCA was loosely ligated with a No. 4-0 silk suture just distal to the arteriotomy, after which the neck wound was rapidly closed.

In 20 animals no therapy was performed after MCA occlusion (Group A: control group). In 48 animals the right cerebral hemisphere was surgically decompressed by means of a craniectomy. Decompressive craniectomy was performed 4 (Group B), 12 (Group C), 24 (Group D), or 36 (Group E) hours after MCA occlusion in 12 animals each. The 68 animals were allocated to the different control or treatment groups (Groups A-E) according to a computer-generated randomization scheme. A bone flap $(0.930 .5 \mathrm{~cm})$ was created in the rat's temporal bone and additional bone was removed under microscopic control down to the floor of the middle fossa using microscissors. The dura covering the frontal, parietal, and temporal lobes was then opened in a large cruciate incision. No cortical resection of infarcted brain was attempted. At the end of the procedure the temporalis muscle and skin flap were adapted and sutured in place.

During the next 7 days all surviving animals were neurologically examined according to a protocol that used an established scoring system first introduced by Bedersen, et al.,[1] and refined by Menzies and colleagues[22] (Table 1); simultaneously each animal's body weight was measured.

\begin{tabular}{|c|c|}
\hline \multicolumn{2}{|c|}{ TABLE 1 } \\
NEUROLOGICAL SCORNG SVSTEM FOR RATS UNDERGONG ENDOVASCULAR \\
MCA OCCLSION太
\end{tabular}

At the end of the 7th day, all animals were reanesthetized with ketamine and xylazine and killed by decapitation. Their brains were rapidly removed and $2-\mathrm{mm}$ brain slices were incubated for 30 minutes in a $4 \%$ solution of 2,3,5 triphenyltetrazolium chloride (TTC) at 37 ̌ C and fixed by immersion in $10 \%$ buffered formalin solution. Normal brain tissue (intact cellular membranes) stains red when exposed to TTC, whereas ischemic tissue turns pink and necrotic tissue turns grayish. Five brain sections per animal were stained with TTC and then photographed.

Following digitization of the photographs, areas of infarction were quantified using a computer and public domain software (IMAGE 1.41; Wayne Rasband, National Insitutes of Health, Bethesda, MD). On each slice the unstained area (ischemic brain) was marked and the infarct volume was calculated according to a thickness of $2 \mathrm{~mm}$ per slice. Measurement and calculation of infarction size were performed twice by two of the authors (C.S. and S.H.) who were blinded to the point of time at which decompressive craniectomy occurred. Assessment of infarction size was made twice, with each side of the brain slices measured separately, and mean values were calculated. To avoid overestimation of the infarct volume, as recently described by Lin, et al.,[20] we related the infarcted volume to the noninfarcted hemisphere to calculate a correcting factor (that is, volume of noninfarcted 
hemisphere/volume of infarcted hemisphere).

\section{Data Analysis}

All statistical analysis were performed with the aid of commercially available software (StatView; Brain Power Inc.) installed on a personal computer (Macintosh Quadra, Apple Computer Inc., Cupertino, CA). For statistical analysis of infarction volume and body weight the unpaired t-test was used; for statistical analysis of neurological score the Kruskal-Wallis test was used; a probability value of less than 0.05 was considered to be significant.

\section{RESULTS}

No statistically significant differences were noted among the five groups for any of the intraoperative physiological parameters. Throughout animal preparation the average body temperature for all animals was $36.9 \check{\mathrm{s} C} \pm 0.5 \check{\mathrm{s} C}$ (mean \pm standard deviation). There were no significant changes in arterial blood gas (PO2 $126 \pm 37 \mathrm{~mm} \mathrm{Hg}$; PCO2 $33 \pm 3.2 \mathrm{~mm} \mathrm{Hg}$; pH $7.4 \pm 0.02$ ) or in hematocrit levels.

\section{Mortality}

Seven of the 20 animals in the control group died, all between 24 and 48 hours after MCA occlusion; therefore, mortality without craniectomy after MCA occlusion was 35\%. None of the animals treated by decompressive craniectomy died during the observation period (mortality rate $0 \%$ ), regardless of whether the procedure had been performed early (after 4 or 12 hours) or late (after 24 or 36 hours). This difference was statistically significant $(\mathrm{p}<0.01)$.

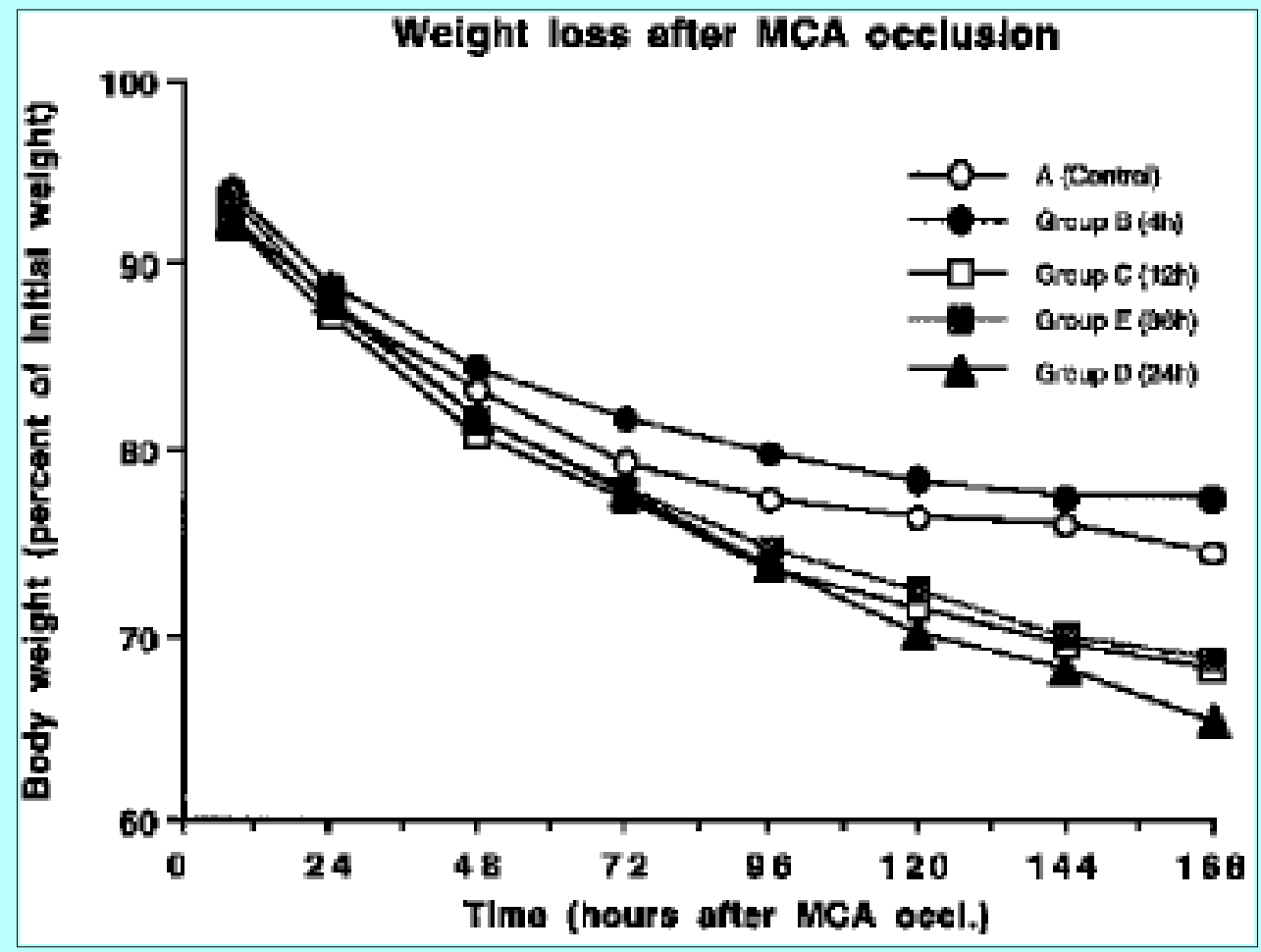

Fig. 1. Graph displaying changes in weight among the control and treatment groups during 168 hours after middle cerebral artery (MCA) occlusion in rats.

\section{Body Weight}

All animals in the study had a decline in body weight. The weight differences at the end of the 7th day 
between the animals in Group A and the animals in Group D was statistically significant $(\mathrm{p}<0.03)$; there was also a significant difference in weight between animals in Group B and Group D ( $p<0.007)$. Figure 1 illustrates our findings regarding these weight changes.

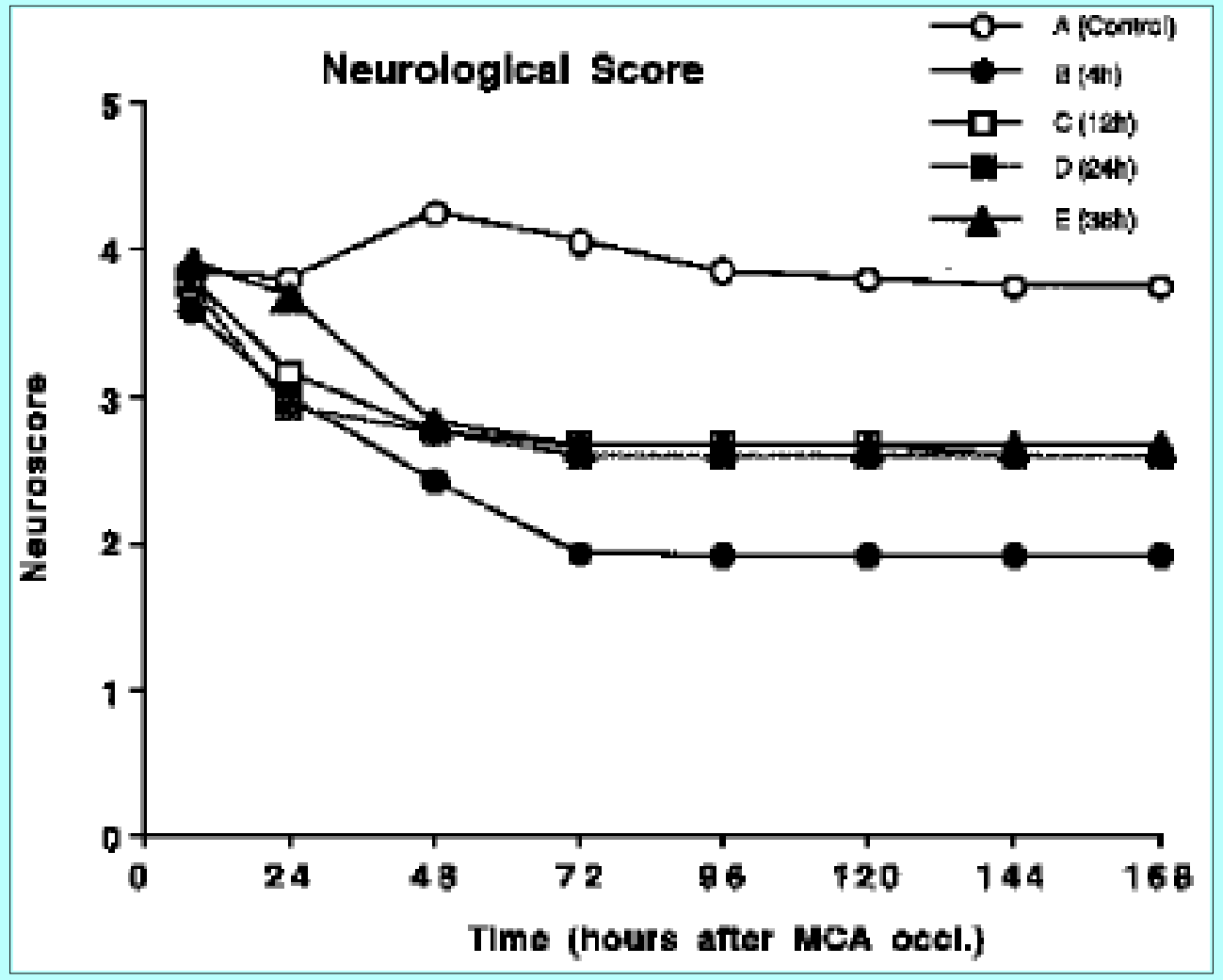

Fig. 2. Graph depicting changes in neurological score (Neuroscore) throughout 168 hours after middle cerebral artery (MCA) occlusion in rats. The differences in neurological behavior between treated and untreated animals were statistically significant; there was also a significant difference between rats treated very early $(B)$ and animals treated late $(C, D$, and $\mathrm{E})$.

\section{Neurological Score}

The average neurological score of the control group (Group A) was 3.7 after 7 days. Animals that underwent a very early craniectomy (Group B) had an average score of 1.9; those that underwent a craniectomy after 12 (Group C), 24 (Group D), or 36 (Group E) hours obtained scores of 2.5, 2.5, and 2.7 , respectively. The difference between treated and untreated animals was statistically significant $(\mathrm{p}<$ $0.0001)$; there was also a significant difference comparing Groups B with Groups C, D, and E ( $<<0.02)$ (Fig. 2). 
TABLE 2

COMPARISON OF INFARCTION YOLUME AFTER ENDOMASCULAR MCA OCCLUSION IN 68 RATS *

\begin{tabular}{|c|c|c|}
\hline Group & Irfanction Volume (mm 3] & $p$ Value \\
\hline Á (20 animals) & $184 \pm 16.9$ & $\begin{array}{l}<0.001 \text { w. Group B十 } \\
<0.12 \text { w. Group o } \\
<0.6 \text { w. Group D } \\
<0.7 \text { w. GroupE }\end{array}$ \\
\hline$B(12$ animals) & $109 \pm 11.25$ & $\begin{array}{l}<0.01 \text { w. Group Ct } \\
<0.002 \text { w. Group D† } \\
<0.007 \text { w. Group } \dagger\end{array}$ \\
\hline $\mathrm{C}(12$ animals $)$ & $150 \pm 9.89$ & $\begin{array}{l}<0.25 \text { w. Group D } \\
<0.25 \text { ws. GroupE }\end{array}$ \\
\hline $\begin{array}{l}\mathrm{D}(12 \text { animals) } \\
\mathrm{E}(12 \text { animals) }\end{array}$ & $\begin{array}{l}169 \pm 13.02 \\
176 \pm 20.0\end{array}$ & $\underset{\text { NA }}{C 0.77 \text { w. GroupE }}$ \\
\hline
\end{tabular}

* Rats in Goup A (control) received no treatment after MCA ocdusion. Rats in the other groups under ment decompressive oraniectomy 4 (Group B), 12 (Group C), 24 (Group D), or 36 (Group E) hours after MCA occlusion. Values of infarction wolume are expressed as the mean 6 standard error of the mean. Abbre viations: $M C A=$ middle ceretral artery; $N A=$ not applicable.

$\dagger$ Statisticaly significant.

\section{Volume of Infarction}

The average volumes of infarction for the different treatment groups are given in Table 2. Early craniectomy performed at 4 hours resulted in significantly smaller infarct volumes than those found in animals subjected to later or no craniectomy. Craniectomy performed at 12 hours or later tended to result in smaller infarctions in comparison to no craniectomy (Fig. 3).

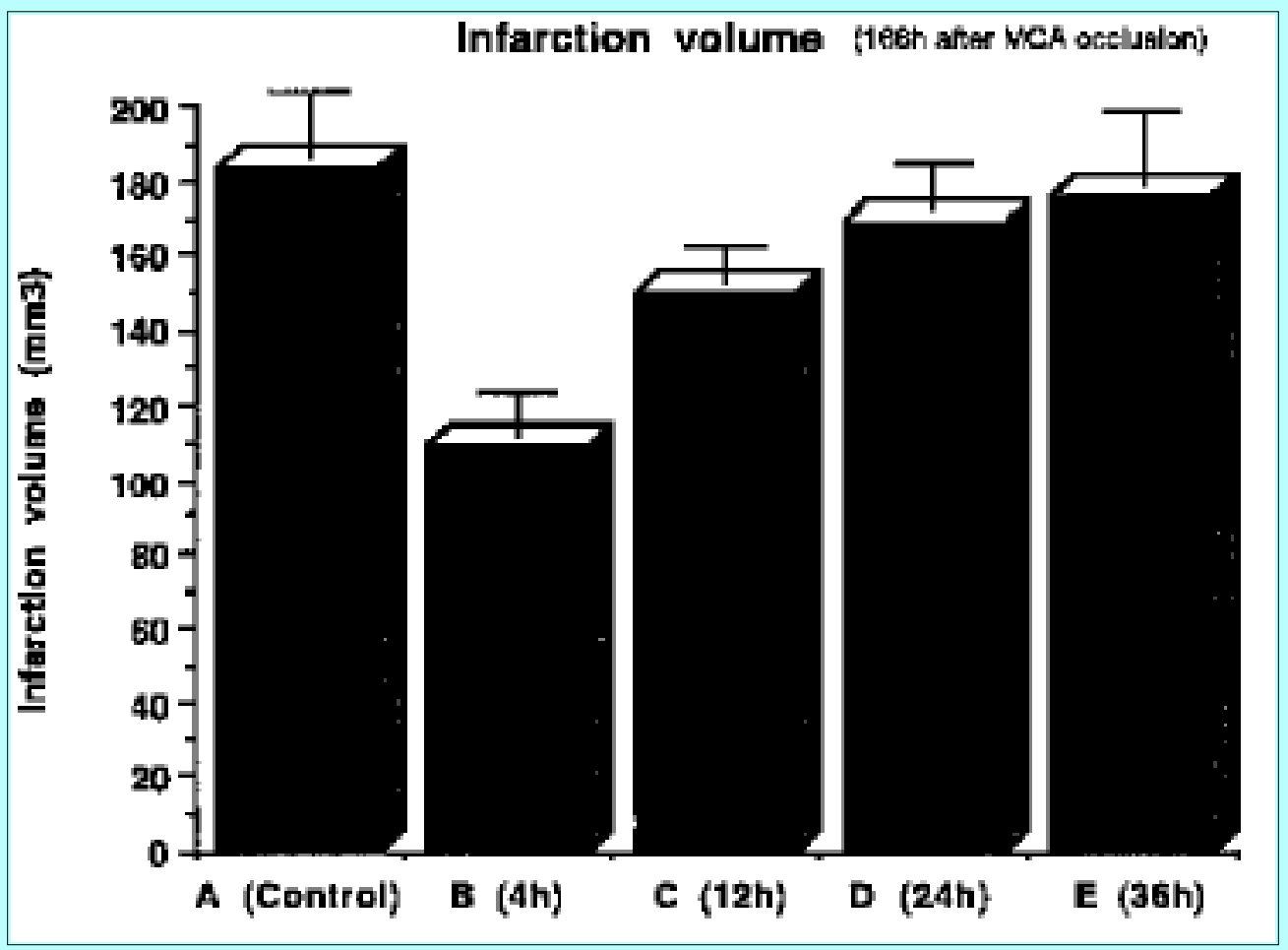

Fig. 3. Graph showing infarction size 168 hours after middle cerebral artery occlusion in rats that received no surgical intervention (A) and those that underwent decompressive craniectomy 4 (B), 12 (C), 24 (D), and 36 (E) hours after vessel occlusion.

Regardless of treatment, all animals suffered an infarction in the basal ganglia, which is supplied by the 
lenticulostriate arteries. There was a trend toward larger and more cortically localized infarctions in the control group and the animals that were treated later (Groups C-E) (Fig. 4).

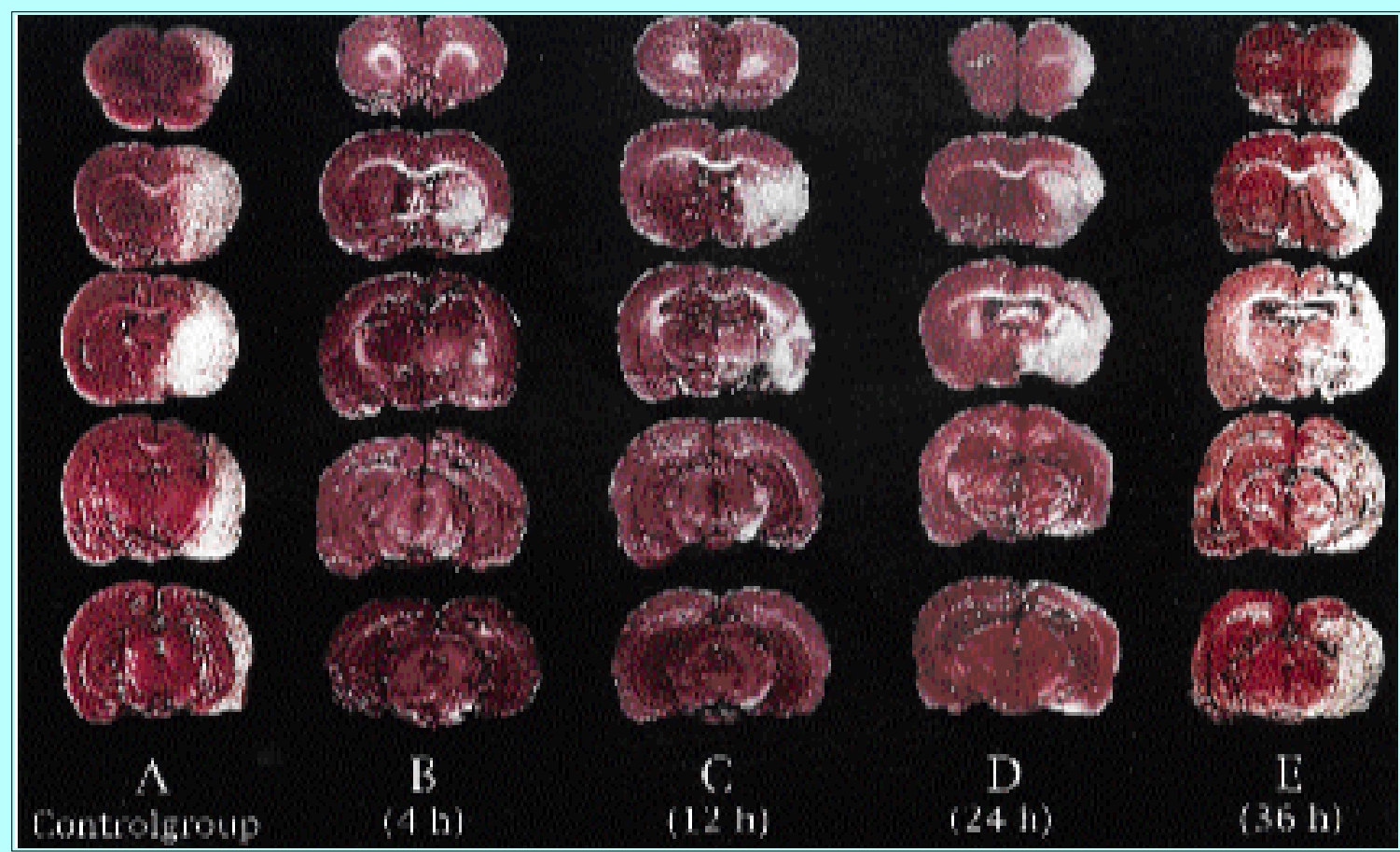

Fig. 4. Brain slices obtained after middle cerebral artery occlusion from rats that received no surgical intervention (A), and rats that underwent decompressive craniectomy 4 (B), 12 (C), 24 (D), and 36 (E) hours after vessel occlusion. Regardless of treatment, all animals suffered a basal ganglia infarction. Note the trend toward more cortically localized infarctions in the animals treated later (Groups C-E). Stain: 2,3,5-triphenyltetrazolium chloride, original magnification x 6 .

\section{DISCUSSION}

Massive unilateral hemispheric infarctions that involve the entire MCA territory occur in $10 \%$ to $15 \%$ of supratentorial infarctions and can be associated with severe brain swelling and death due to brain herniation.[26] This "malignant" hemispheric infarction of the complete MCA territory or an even greater area (ACA and, rarely, posterior cerebral artery (PCA) territories) is usually due to embolic ICA or proximal MCA segment (M1) occlusion with insufficient collateral flow and development of early brain edema. Mortality rates for unselected groups of patients with MCA infarctions of different sizes and locations are $30 \%$ to $66 \% ;[17,35,38]$ Hacke, et al.,[13] reported a mortality rate of $80 \%$ in patients who developed a malignant hemispheric infarction. For an early and, probably, most effective treatment, the malignant character of an ischemic lesion has to be recognized during the 1st hours after onset of symptoms. Knowledge of the natural history of malignant hemispheric infarction is, therefore, a precondition for aggressive therapeutic options such as decompressive surgery. Surprisingly, the literature contains few studies regarding the prognosis in patients with malignant hemispheric infarction. Clinical parameters, such as coma, pupillary areactivity, and dense hemiplegia, are not really early indicators but are rather signs of a malignant MCA infarct. Computerized tomography (CT) criteria seem to have a higher prognostic value. In a prospective study of 53 patients with MCA infarction, von Kummer, et al.,[37] showed that if parenchymal hypodensity on CT scans covers more than $50 \%$ of the MCA territory, "malignant" brain edema will occur with a likelihood of $85 \%$. In the European Cooperative Acute Stroke study, death due to herniation following massive ischemic brain edema was 
the most frequent cause of death during the 1 st 7 days and was found in $7.3 \%$ of the right pulmonary artery-treated group and in $4.9 \%$ of the placebo group.[12] Prognosis is correlated with the severity of the initial neurological deficit. Hemiplegia, forced eye deviation, and decreased level of consciousness indicate a poor outcome.[13] Age may be a prognostic factor in that younger, less atrophic brains are less able to accommodate swelling than are older brains with generalized atrophy.[19]

In most cases, aggressive treatment with intubation, artificial ventilation, and antiedema agents cannot prevent fatal herniation. In the management of such cases, decompressive craniectomy has been recommended and indeed may be an appropriate, lifesaving procedure.[8,16,19,30,32,39]

The idea of removing a segment of the cranial vault to relieve intracranial pressure is one of the oldest neurosurgical concepts. An early indication for the procedure was palliation for increased intracranial pressure from tumors that were not localizable by the available neurodiagnostic techniques. In his landmark article of 1905, Harvey Cushing[7] described the technique of cranial decompression by a subtemporal approach. In patients with massive supratentorial edema caused by head trauma, however, the beneficial effect of decompressive craniectomy is still debatable, because decompression may result in edema progression. $[6,14]$ An analogous situation in the posterior fossa caused by ischemic necrosis of a cerebellar hemisphere is now widely accepted as a surgical emergency requiring surgical decompression with removal of infarcted brain.[15,31] There is some controversy over whether the infarction should be resected or not.[11,16,30] However, because in acute stroke the margins of the infarction are poorly defined and differentiation between definitely damaged tissue and the ischemic penumbra is not possible, we did not remove the ischemic tissue in this experimental study.

In anecdotal reports decompressive craniectomy was found to be effective for treatment of massive edema related to supratentorial stroke.[16,17,19,30,39] In a small series of five patients, Kondziolka and Fazl[19] found craniectomy to be a life-saving therapy in stroke patients. Rengachary and colleagues[30] reported three cases of patients with large hemispheric infarctions who survived after craniectomy; however, in two of them a severe focal neurological deficit persisted. Kalia and Yonas[17] reported on four patients who were treated by decompressive craniectomy plus resection of nonviable brain; all survived with a relatively good clinical outcome. All previous studies were anecdotal and retrospective. A recently performed prospective study of 53 patients with malignant hemispheric infarction showed the beneficial effects of decompressive hemicraniectomy on mortality and morbidity, supporting the notion that aggressive surgical therapy should be started as soon as possible.[32] However, the optimal time at which decompressive surgery should be performed still remains debatable.

Thus far, few experimental findings have been published on the usefulness of decompressive craniectomy in the treatment of acute stroke.[10] The main reason for this lack of experimental studies is probably that one of the most commonly used models of focal cerebral ischemia, first described by Tamura, et al.,[36] requires a craniectomy to occlude the MCA. This model, therefore, does not allow us to compare the therapeutic effects of craniectomy with a control group. Embolic models, on the other hand, do not require a craniectomy, but infarction size is extremely variable and therapeutic effects are difficult to quantify unless large numbers of animals are used. The lack of an appropriate model of focal cerebral ischemia has thus prevented a systematic experimental investigation of the therapeutic effect of decompressive craniectomy in large cerebral infarction. A model of endovascular occlusion, first described by Koizumi, et al.,[18] and modified by others,[24,40] simulates MCA occlusion nearly perfectly in humans and does not require any surgical manipulation of the skull. In its correct position the intraluminal suture reaches the proximal segment of the ACA. At this point the suture has blocked the 
origin of the MCA, occluding all sources of collateral blood flow from the ICA, ACA, and PCA, resulting in large hemispheric ischemia lesions.[40]

The mortality rate in our experimental study was $0 \%$ for all animals treated by decompressive craniectomy versus $35 \%$ in the control group. All of the animals that died in the control group died between 24 and 48 hours after vessel occlusion.

We wondered about the lack of deaths in the 36-hour group; however, we attribute this to the beneficial effect of decompressive craniectomy as a livesaving procedure, even 36 hours after MCA occlusion. Because all 68 animals were randomly allocated to the five different treatment groups (Groups A-E) there was no selection. This reduction in mortality likely results from preventing uncal herniation by converting the cranium from a "closed" cavity into an "open" one. The most striking observation in our current study was the reduced infarction size and improved neurological score in the animals that were treated very early (Group B). There was also a difference in infarction volume in Group C (12 hours) when compared with Groups D to E and the control group (Group A), respectively (Fig. 3). Craniectomy performed later than 4 hours after vessel occlusion was lifesaving but improved neurological outcome to a lesser extent than early craniectomy. Compared to untreated animals (Group A), the neurological score and behavior of all treated animals were significantly better (Fig. 2). The continuous decline in body weight of $35 \%$ in Groups $\mathrm{C}$ to $\mathrm{E}$ might be due to the additional burden of a second course of anesthetic medication and surgery, whereas animals in Group A and B, anesthetized only once, had a decline in body weight of only $25 \%$ (Fig. 1). For technical reasons, it was not possible to quantify infarction size in those untreated animals of Group A that died as a result of herniation before Day 7 because TTC staining is not effective so many hours after death. Considering that these infarcts were the largest in the whole group, the real difference in infarction size between treated and untreated animals is even greater than was calculated. The same is probably true for body weight. Our data analysis in Group A is more or less a "best-case" analysis: if the data for animals that died early had been available, the differences between treated and untreated animals would have been larger.

In our study infarction volumes and neurological scores in the treated group were higher compared to the results of a recently published study on the effect of decompressive craniectomy in rats.[10] In that study, an average infarction volume of $160 \pm 21 \mathrm{~mm}$ cubed was reported in the untreated control group, $26 \pm 12$ $\mathrm{mm}$ cubed in a group treated by early craniectomy 1 hour after MCA occlusion, and $59 \pm 15 \mathrm{~mm}$ cubed in a group treated 24 hours after MCA occlusion, respectively. This difference compared to the results of our present study may be due to a different method of endovascular MCA occlusion, one without a silicone-coated suture.[40] In the present study we used the method described by Koizumi, et al.,[18] who used a silicone-coated endovascular thread to increase the success rate of complete MCA occlusion. Comparison of absolute values is therefore difficult, but the tendency toward the effectiveness of aggressive decompressive surgery early after onset of stroke is still obvious.

Why does reduced infarction size in early treated animals result in improved neurological performance? In this study intracranial pressure, perfusion, and temperature were not measured so that we could maintain a closed-skull preparation. Because we sutured the temporalis muscle and the skin flap in place after decompressive surgery, we do not believe that a "cooling effect" of the brain played a major role. However, we cannot exclude the possibility that the craniectomy lowered brain temperature, which could have protected the brain from ischemic damage. There are several arguments, however, that favor a major role for intracranial pressure. Ischemic cerebral infarction associated with extensive edema and a marked elevation of intracranial pressure may cause ischemia of neighboring brain tissue and thus lead to 
further infarction. Decompressive craniectomy may interrupt this vicious circle by decreasing the pressure of CSF and brain tissue.[14] This may increase cerebral perfusion pressure and optimize retrograde perfusion of MCA branches via leptomeningeal collateral vessels; functionally compromised but viable brain may thus be able to survive.

All of the animals in the study, whether they were treated or not, suffered an ischemic infarction in the territory supplied by the lenticulostriate arteries (Fig. 4), which are functional end arteries. This is the area of the MCA territory in which cerebral blood flow is most suppressed when using the endovascular model of cerebral ischemia.[21] In general, this is equivalent to M1 occlusions in humans: patients with an excellent leptomeningeal collateral blood supply usually suffer only a subcortical striatocapsular infarction.[9] This parallel between human stroke and our animal model provides a further argument for better perfused leptomeningeal collateral vessels as the main reason for reduced infarction size after decompressive craniectomy.

Hemicraniectomy is technically a simple procedure that could be performed in any community hospital with minimal ancillary support: a major advantage when considering that cerebrovascular disease is the most common neurological cause of death.

One problem facing the clinician is to determine the length of time that should be allowed for conservative therapy before considering decompressive craniectomy. It is widely accepted that decompressive craniectomy should not be postponed so long that irreversible brainstem changes, such as Duret hemorrhages, occur; however, the exact point of time when craniectomy would be most beneficial is unclear. Shaw and colleagues[34] suggested in 1959 that death in humans during the 1st week following infarction was directly related to vasogenic brain edema, which reaches a peak within 2 to 5 days. Most patients with massive edema develop brain herniation within 48 hours after the onset of ischemia.[2] This time course does not exactly parallel that of rats, in which the peak of edema usually occurs within the first 24 to 48 hours.[27] Therefore our results (and those of experimental studies in general) cannot provide the exact answer to the question regarding optimal timing of craniectomy in humans. We systematically performed decompressive surgery at different points of time, that is, at 4, 12, 24, and 36 hours after vessel occlusion. The differences between the very early and late treated groups were statistically significant and there was a trend toward larger cortical infarcts in animals that underwent a late craniectomy (after 24 and 36 hours). The results obtained in rats cannot be directly transferred to humans, as the ratio of brain volume/skull volume in rats differs from that in humans and (as mentioned above) the quality of leptomeningeal collateral also differs substantially. Caution should always be exercised in extrapolating data from an animal model to humans. The exact time window for decompressive craniectomy in humans as an alternative to medical therapy has to be evaluated in controlled patient studies. Patient studies are also important with respect to neurological morbidity in humans, something that cannot very well be evaluated in rats. The end point of morbidity in rats alone is certainly not as important as the end point of significant morbidity in human patients.

Integration of clinical examination with early CT findings[37] and new imaging techniques, such as perfusion- and diffusion-weighted magnetic resonance imaging,[23] could prove valuable in determining the clinical significance of infarction early after onset, thereby allowing us to gauge aggressive treatment forms, such as craniectomy, before life-threatening brain swelling and herniation occur. Investigation of prognostic factors in future randomized studies is required to establish the criteria by which patients are to be selected for craniectomy. 


\section{CONCLUSIONS}

Our results suggest that decompressive craniectomy for cerebral ischemia not only reduces mortality but also significantly improves outcome and reduces infarction size, especially when performed early after vessel occlusion. Craniectomy later than 4 hours after vessel occlusion can be lifesaving but does not improve neurological outcome or reduce infarction size as much as very early craniectomy. This experimental support for an aggressive prompt therapeutic approach indicates that a controlled study of decompressive craniectomy in selected patients with acute ICA or MCA occlusion would be worthwhile. By performing decompressive craniectomy neurosurgeons will play a major role in the management of stroke patients.

\section{Acknowledgment}

This work contains parts of the thesis by Christian Staff.

\section{References}

1. Bedersen JB, Pitts LH, Tsuji M, et al: Rat middle cerebral artery occlusion: evaluation of the model and development of a neurologic examination. Stroke 17:472-476, 1986

2. Bounds JV, Wiebers DO, Whisnant JP, et al: Mechanisms and timing of deaths from cerebral infarction. Stroke 12:474-477, 1981

3. Camarata PJ, Heros RC, Latchaw RE: "Brain attack;" the rationale for treating stroke as a medical emergency. Neurosurgery 34:144-158, 1994

4. Chen HJ, Lee TC, Wei CP: Treatment of cerebellar infarction by decompressive suboccipital craniectomy. Stroke 23:957-961, 1992

5. Clarke E, Harris P: Thrombosis of the internal carotid artery. Simulating an intracranial space-occupying lesion. Lancet 1:1085-1089, 1958

6. Cooper PR, Hagler H, Clark WK, et al: Enhancement of experimental cerebral edema after decompressive craniectomy: implications for the management of severe head injuries. Neurosurgery 4:296-300, 1979

7. Cushing H: The establishment of cerebral hernia as a decompressive measure for inacessible brain tumors: with the description of intermuscular methods of making the bone defect in temporal and occipital regions. Surg Gynecol Obstet 1:297-314, 1905

8. Delashaw JB, Broaddus WC, Kassell NF, et al: Treatment of right hemispheric cerebral infarction by hemicraniectomy. Stroke 21:874-881, 1990

9. Forsting M, Krieger D, von Kummer R, et al: The prognostic value of collateral blood supply in acute middle cerebral artery occlusion, in DelZoppo GJ, Mori E, Hacke W (eds): Proceedings of the 2nd International Symposium on Thrombolytic Therapy in Acute Ischemic Stroke. Berlin: Springer-Verlag, 1993, pp 160-167

10. Forsting M, Reith W, Schäbitz WR, et al: Decompressive craniectomy for cerebral infarction. An experimental study in rats. Stroke 26:259-264, 1995 
11. Greenwood J Jr: Acute brain infarctions with high intracranial pressure: surgical indications. John Hopkins Med J 122:254-260, 1968

12. Hacke W, Kaste M, Fieschi C, et al: Intravenous thrombolysis with recombinant tissue plasminogen activator for acute hemispheric stroke. The European Cooperative Acute Stroke Study (ECASS). JAMA 274:1017-1025, 1995

13. Hacke W, Schwab S, De Georgia M: Intensive care of acute ischemic stroke. Cerebrovasc Dis 4:385-392, 1994

14. Hatashita S, Hoff JT: The effect of craniectomy on the biomechanics of normal brain. J Neurosurg 67:573-578, 1987

15. Heros RC: Surgical treatment of cerebellar infarction. Editorial. Stroke 23:937-938, 1992

16. Ivamoto HS, Numoto M, Donaghy RMP: Surgical decompression for cerebral and cerebellar infarcts. Stroke 5:365-370, 1975

17. Kalia KK, Yonas H: An aggressive approach to massive middle cerebral artery infarction. Arch Neurol 50:1293-1297, 1993

18. Koizumi J, Yoshida Y, Nakazawa T, et al: Experimental studies of ischemic brain edema: 1. A new experimental model of cerebral embolism in rats in which recirculation can be introduced in the ischemic area. Jpn J Stroke 8:1-8, 1986

19. Kondziolka D, Fazl M: Functional recovery after decompressive craniectomy for cerebral infarction. Neurosurgery 23:143-147, 1988

20. Lin TN, He YY, Wu G, et al: Effect of brain edema on infarct volume in a focal cerebral ischemia model in rats. Stroke 24:117-121, 1993

21. Memezawa H, Smith ML, Siesjö BK: Penumbral tissues salvaged by reperfusion following middle cerebral artery occlusion in rats. Stroke 23:552-559, 1992

22. Menzies SA, Hoff JT, Betz LA: Middle cerebral artery occlusion in rats: a neurological and pathological evaluation of a reproducible model. Neurosurgery 31:100-107, 1992

23. Mintorovitch J, Moseley ME, Chileuitt L, et al: Comparison of diffusion- and T2-weighted MRI for the early detection of cerebral ischemia and reperfusion in rats. Magn Reson Med 18:39-50, 1991

24. Nagasawa H, Kogure K: Correlation between cerebral blood flow and histologic changes in a new rat model of middle cerebral artery occlusion. Stroke 20:1037-1043, 1989

25. National Institute of Neurological Disorders and Stroke rt-PA Stroke Study Group: Tissue plasminogen activator for acute ischemic stroke. N Engl J Med 333:1581-1587, 1995

26. Ng LKY, Nimmannitya J: Massive cerebral infarction with severe brain swelling. A clinicopathological study. Stroke 1:158-163, 1970

27. O'Brien MD, Waltz AG, Jordan MM: Ischemic cerebral edema. Distribution of water in brains of cats after occlusion of the middle cerebral artery. Arch Neurol 30:456-460, 1974 
28. Ojemann RG, Heros R, Crowell RM: Surgical Management of Cerebrovascular Disease, ed 2. Baltimore: Williams \& Wilkins, 1987, p 143

29. Olsen TS: Outcome following occlusion of the middle cerebral artery. Acta Neurol Scand 83:254-258, 1991

30. Rengachary SS, Batnitzky S, Morantz RA, et al: Hemicraniectomy for acute massive cerebral infarction. Neurosurgery 8:321-328, 1981

31. Rieke K, Krieger D, Adams HP, et al: Therapeutic strategies in space-occupying cerebellar infarction based on clinical, neuroradiological and neurophysiological data. Cerebrovasc Dis 3:45-55, 1993

32. Rieke K, Schwab S, Krieger D, et al: Decompressive surgery in space-occupying hemispheric infarction: results of an open prospective trial. Crit Care Med 23:1576-1587, 1995

33. Selman WR, Spetzler RF: Therapeutics for focal cerebral ischemia. Neurosurgery 6:446-452, 1980

34. Shaw CM, Alvord EC Jr, Berry GR: Swelling of the brain following ischemic infarction with arterial occlusion. Arch Neurol 1:161-177, 1959

35. Steiger HJ: Outcome of acute supratentorial cerebral infarction in patients under 60. Development of a prognostic grading system. Acta Neurochir 111:73-79, 1991

36. Tamura A, Graham DI, McCulloch J, et al: Focal cerebral ischaemia in the rat: 1. Description of technique and early neuropathological consequences following middle cerebral artery occlusion. J Cereb Blood Flow Metab 1:53-60, 1981

37. von Kummer R, Meyding-Lamadé U, Forsting M, et al: Sensitivity and prognostic value of early CT in occlusion of the middle cerebral artery trunk. AJNR 15:9-15, 1994

38. Yoshimoto T, Ogawa A, Seki H, et al: Clinical course of acute middle cerebral artery occlusion. J Neurosurg 65:326-330, 1986

39. Young PH, Smith KR Jr, Dunn RC: Surgical decompression after cerebral hemispheric stroke: indication and patient selection. South Med J 75:473-475, 1982

40. Zea Longa E, Weinstein PR, Carlson S, et al: Reversible middle cerebral artery occlusion without craniectomy in rats. Stroke 20:84-91, 1989

Manuscript received March 7, 1996.

Accepted in final form June 19, 1996.

Address reprint requests to: Arnd Doerfler, M.D., Department of Neuroradiology, University of Heidelberg Medical School, Im Neuenheimer Feld 400, D-69120 Heidelberg, Germany. 